\title{
DAS NEUROOTOLOGISCHE STUDIUM ÜBER DIE LÄSION DES KLEINHIRNWURMS
}

\author{
Eiji SaKata
}

\section{EINLEITUNG}

Die klinische Diagnose der Kleinhirnstörungen, besonders der Störungen im Wurm war bisher sehr schwer, und deshalb sind deren berichteten Fälle hauptsächlich durch Sezierung klargelegt und zwar sind sie immer vaskuläre, pathologische Veränderungen.

Im allgemeinen ist das Hämatom am Kleinhirn unter den idiopathischen Hämatoma im Hirn verhältnismässig selten, und ausserdem ist dessen Prognose wegen des Bruchs des Hämatoms in Ventriculus quartus oder Hirnstammkompressionssyndrome des Hämatoms ausserordentlich ungünstig. Jedoch in letzter Zeit, dank der Bemühungen der verschiedenen Forscher, vermehren sich die Berichte über die durch die Operation geheilten Fälle.

Wir haben neulich insgesammt 4 Fälle erfahren, bei 2 Fällen davon wurde das Hämatom am Wurm festgestellt und exstirpiert, unnd bei anderen zwei Fällen wurde es nicht festgestellt aber klinisch war das Symptom äusserst ähnlich.

Wir haben ferner bei diesen Fällen eine sehr eigenartige Blickstörung, heftigen Lagerungsschwindel sowie Lagerungsnystagmus beobachten können.

Auf Grund unserer Untersuchung sind wir überzeugt, dass unter den obengenannten eigenartigen Störungen der Augenbewegungen die erstere auf der Störung des mittlen Teils am Wurm, der hauptsächlich aus Folium und Tuber besteht, und die letztere auf der Unterwurmstörung beruht. Wir möchten hier von diesen Fällen benachrichtigen und um Ihre Kritik bitten.

\section{FÄLLE}

Fall I: S.N., 28 Jahre, männlich, Bauer, dextromanuell.

Hauptsymptome: Schwindelanfall, Anfallsweise Hinterkopfschmerzen, Aufstehstörung, Sprachstörung, Lähmungsgefühl an der rechten Hand, allgemeiner Krampfanfall und Doppelsehen.

Familiengeschichte: Sein Vater hat Ohnmachtsanfallanamnese und die Mutter subarachnoidale Bultungsanamnese.

Anamnese: nichts besonderes.

Status praesens: Seit etwa 3 Jahren fühlt sich der Patient an der rechten Hand Lähmung, wenn er beschäftig ist. Ungefähr vor zwei Jahren (im Herbst) wollte er sich mit plötzlichem Drehschwindel erbrechen und fiel in Ohnmacht. Allgemeiner tonischer Krampf

Aus der Hals-Nasen-Ohrenklinik der medizinischen Fakultät an der Universität Juntendo, Tokyo 
und Supraduktionszustand der beiden Augen dauerten ein paar Minuten. Vor acht Monaten bei Einstellung des Fernsehenbildes hatte der Kranke plötzlich einen Drehschwindel in Richtung des Uhrzeigers mit Brechreiz und wurde ohnmächtig. Auch allgemeiner, tetanisch-klonischer Krampf bekam er.

In einer Irrenheilanstalt wurde er untersucht und nach der EEG wurde er als ,Epilepsie" diagnostiziert. Er nahm das Krampfmittel ein und seitdem keine obengenannten Anfälle. Seit etwa 4 Monaten fühlt er sich oft die Lähmung der rechten Hand und sie wird immer stärker.

Eines Morgens vor drei und halbem Monaten bekam er bei Ausleerung plötzlich sehr heftigen Hinterkopfschmerz und wieder Schwindel, ferner an beiden Unterglieder fühlte er sich ganz kraftlos. Er konnte nicht aufstehen, gleichzeitig wurde das Lähmungsgefühl an beiden Armen, besonders dem rechten Arm grösser. Er hatte eine schwere Zunge, doch keine Bewusstseinsstörung und Krämpfe. Wegen des subarachnoidalen Blutungsverdacht wurde die Lumbalpunktion durchgeführt: Anfangsdruck $210 \mathrm{~mm} \mathrm{H}_{2} \mathrm{O}$, blutig.

Hauptresultate der Prüfungen: klares Bewusstsein, Augenhintergurnd war ein bisschen ödematös, kein Befund, der das Dasein des Hippel-Lindau-Angiomas feststellt und am ganzen Körper keine besondere Veränderung ( 2 Tage nach dem Krankenhauseitritt erschien die Nackenstarre). Keine Hirnnervensymptome. Als Pyramidenzeichen war die Steigerung des Sehnenreflxes an beiden Untergliedern erkennbar(rechts $>$ links). Fussklonus wurde beobachtet, aber kein pathologischer Reflex. Der Patient klagte über Lähmungsgefühl an dem rechten Arm, aber objektiv war keine Differenz zwischen beiden Armen zu beobachten.

Beim doppelseitigen Finger-Nasen-Versuch war der Patient ungeschickt. Kein Extrapyramidenzeichen war sichtbar. Keine auffallende Veränderung von CAG war erkennbar.

Unter dem Zustand des Geraderichtungfernblicks wurde der kurzperiodisch unregelmässig richtungsalternierende horizontale Nystagmus beobachtet und bei der Lage- und Lagerungsprüfung entstand der feinschlägige vertikale Nystagmus mit Schwindelgefühl. Entstehungshemmung des optokinetischen Nysatgmus (rechts<links). Eine bemerkenswerte Blickfolgebewegungsstörung wurde durch die Blickfolgebewegungsprüfung klargelegt. Das Resultat des vertikalen Schreibversuchs war gut.

Der Kranke wurde sofort ins Krankenhaus aufgenommen. Nach dem Eintritt ins Krankenhaus wurden der Hinterkopfschmerz, Brechreiz und Erbrechen heftiger. Ungefähr vom 7. Tag an war die Stauungspapille erkennbar. Verspätetes Urineren und Tremor am linken Arm erschienen. Bei PEG wurde die symmetrische Vergrösserung der doppelseitigen Gehirnkammer und des Ventriculus tertius festgestellt. Durch VAG waren im lateralen Bild das Hinausdrängen der Arteria basialis nach Vorderseite sowie das Hinaufdrängen der Arteria c. posterior erkennbar. Durch die Myodil-Ventrikulographie wurde im lateralen Bild der Schattendefekt im hinteren Teil des Ventriculus quartus beobachtet. Bei EEG waren die langsamen Wellen von 3-4 cps im rechten Hinterkopf sichtbar, aber 
keine Stachel-Welle (spike wave).

Operationsbefunde: Der Verfasser entschloss sich, wegen des Verdachts auf dem Tumor im Ventriculus quartus und der Blutung daraus die Kraniotomie durchzuführen.

In sitzender Lage wurde die suboccipitale Dekompression durchgeführt.

Der intrakranielle Druck war bemerkenswert gesteigert. Ein leichter Vorfall der seitigen Kleinhirnmandel und eine deutliche rückwärtige Ausstülpung des Wurms waren sichtbar. Der Wurm wurde aufgeschnitten. Von der Stelle etwa $5 \mathrm{~mm}$ tief unter der

a) Spontan-und Provokations-Nystagmus

Spontan-u. Blicknystagmus

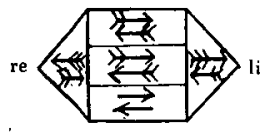

Sitzend

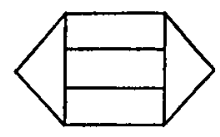

Liegend

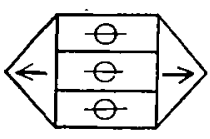

Sitzend
unter der Frenzel - Leuchtbrille

Lageprüung
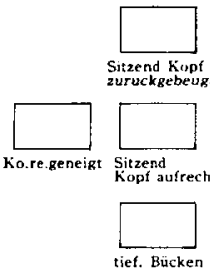

Lagerungsprüfung
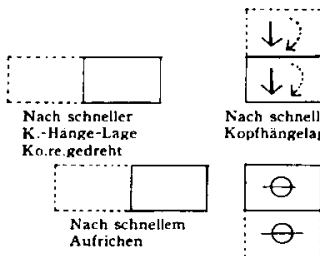
Nach schneller

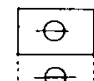

$\theta$

Nach Schnellem
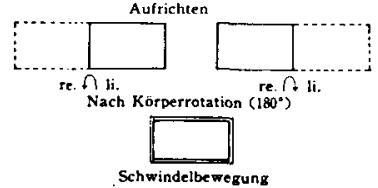

Zahllos !

Schwindelgefühl begleitet
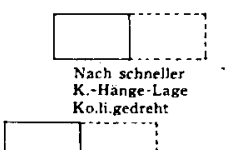

Nach schnellem

Aufrichten

Schwindelgefühl begleitet!

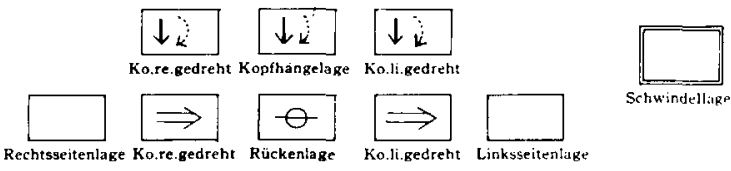

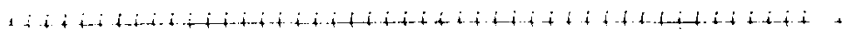

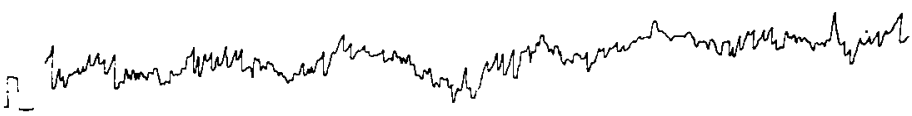

(b)

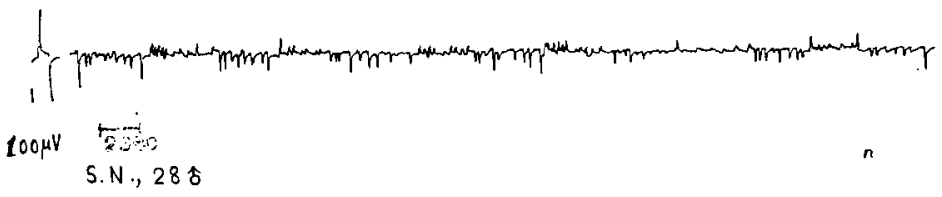

Equilibrium Res Suppl. 1 
(c)

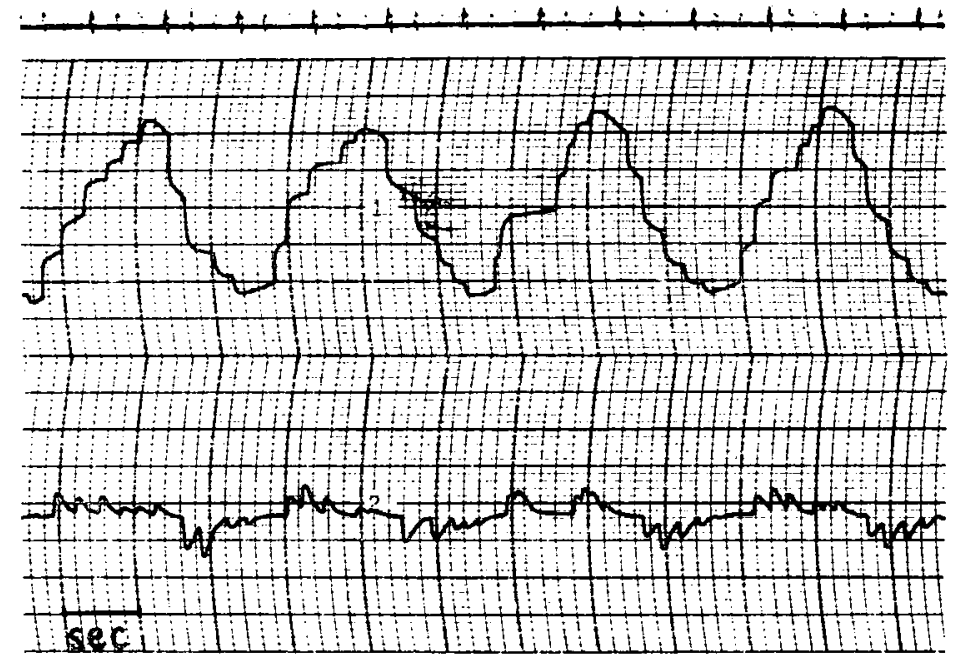

S.N.

Abb. 1: S.N., 28 今

a) Unter dem Zustand des Geraderichtungfernblicks wurde der kurzperiodisch unreglmässig richtungsalternierende horizontale Nystagmus beobachtet und bei Lage-und Lagerungsprüfung entstand der feinschlägige vertikale Nystagmus mit Schwindelgefühl.

b) Die ENG-Registrieung des obengenannten Nystagmus. Die obere Kurve zeigt Augendeviation selbst und die untere die Geschwindigkeit der langsamen Phase.

c) Eine bemerkenswerte Verfolgungsmotorikstörung wurde durch die Prüfung der Blickfolgebewegung klargelegt.

Oberfläche an wurden Cysten gebildet und darin war ein Blutgerinnsel erkennbar. Kein Tumor befand sich dort. Bei den patho-histologischen Prüfungen der Cystenwand waren keine Befunde erhältlich, die das Dasein des Gefässschwamms bestätigen.

Betrachtung: Während des ganzen Behandlungsverlaufs von dem Krankenhauseintritt bis Operation war der Befund des Spontan- und Provokations-Nystagmus immer konstant beobachtbar.

Nach der Operation verschwinden die Kopfschmerzen und Schwindelanfall. Gang des Patienten ist ataktisch, doch möglich.

Im Vergleich mit dem Zustand vor der Operation ist das Symptom der linken Kleinhirnhemisphäre noch beträchtlicher geworden. Es ist mit Recht zu vermuten, dass das auf dem Haematom, die durch den Bruch des Haemoangioms im Bereich der Arteria c. superior entstand (obgleich sie patho-histologisch nicht festgestellt wurde) beruht. (s. Bilder 1-a, b, c)

Fall II : T.F. 32 Jahre, männl. Arbeiter, dextromanuell.

Hauptsymptome: Hinterkopfschmerz, Kopfschwergefühl, Schulterspannung (rechts >links),

Lähmungsgefühl der rechten Hand, Schwindel, Oscillopsie.

Familiengeschichte: nichts besonders

Anamnese: nichts besonders ausser Myopie, Astigmatismus, rechter Strabismus convergens. 
Status praesens: Seit ungefähr 5 Jahren fühlt er sich eine plötzliche Lähmung an der rechten Hand, deren Empfindung wird stumpf und gleichzeitig fühlt er sich, dass die Kraft der rechten Hand verloren geht. Diese Erscheinungen dauern oft eine Stunde lang. Seit einem Jahr bringt dieser Anfall auch einen heftigen Hinterkopfschmerz, Brechreiz und Erbrechen mit sich und der Patient litt daunter immer 2-3 Stunden.

Vor zwei Wochen erhielt er mit einem starken Hinterkopfschmerz noch Schwindelanfall, Brechreiz, Erbrechen, Lähmungsgefühl an den beiden Armen, und eine Woche lang danach konnte er nicht gehen und lag im Bett, dann allmählich erholte er sich. Seit diesem Anfall erscheint horizontale Oscillopsie.

Prüfungsresultate: Blutdruck 102-60, Myopie, Astigmatismus und rechter Strabismus convergens sind zu erkennen. Der Befund des Augenhintergrundes ist nicht besonders ungewöhnilch. Hypästhesie des rechten Gesichts ist bemerkt. Griffstärke, rechts $<$ links. Keine Steigerung des Sehnenreflexes und kein pathologischer Reflex sind erkennbar. Kein Kleinhirnsymptom. Der Nystagmus ist sehr eigenartig und ist ein horizontaler Nystagmus, dessen Richtung sich beim Geraderichtungsfernblicken nach rechts und links unregelmässig verändert. Aber unter Benutzung der Leuchtbrille verschwindet er. Bei der Lageprüfung erscheint der richtungsalternierende aufwärtige Nystagmus,

\section{a) Spontan-und Provokation-Nystagmus}

Spontan-u. Blicknystagmus

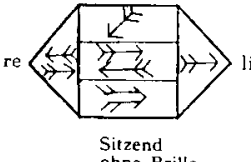

Lageprüfung
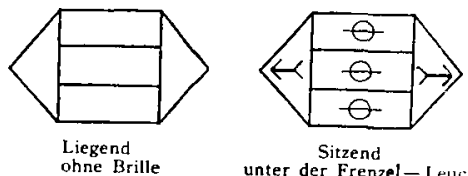

Sitzend
unter der Frenzel- Leuchtbrille

Schwindelgefühl begleitet
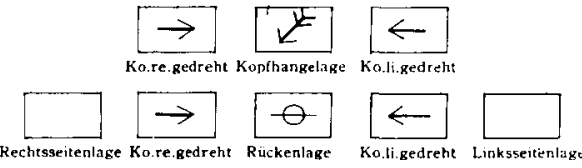

S.hwindellige

ca. 30mal

Schwindelgefühl begleitet ."

Lagerungsprüfung
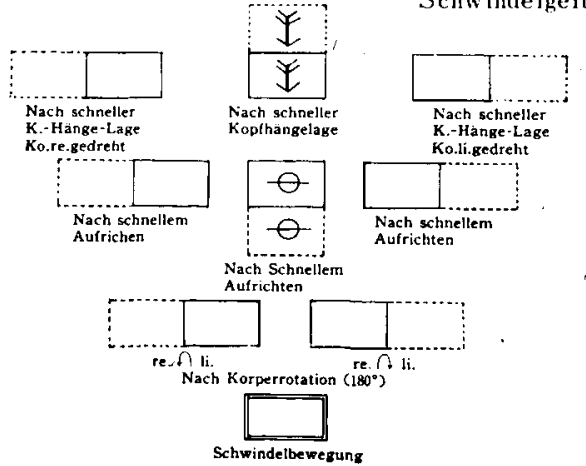

Equilibrium Res Suppl. 1 
(b)

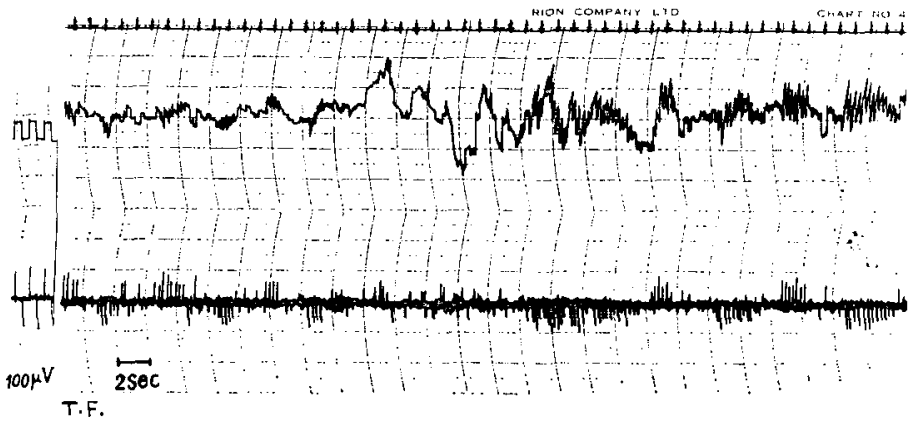

(c)

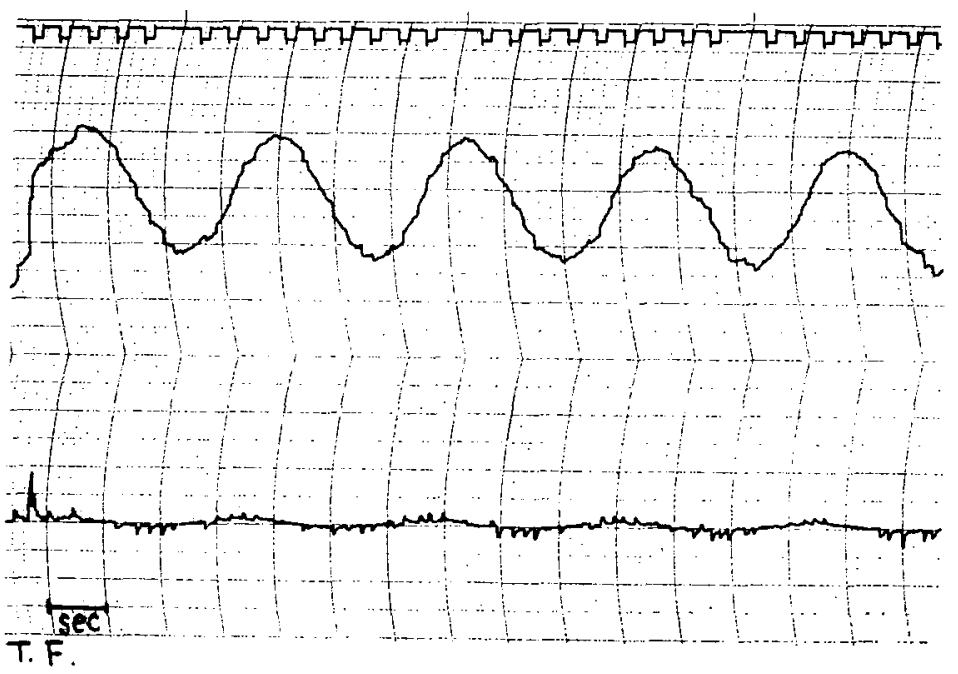

Abb. 2 : T.F., 32 今

a) Der Nystagmus ist sehr eigenartig und ist ein horizontaler Nystagmus, dessen Richtung sich beim Geraderichtungsfernblicken nach rechts und links unregelmässig verändert. Aber unter Benutzung der Leuchtbrille verschwindet er. Bei der Lageprüfung erscheint der richtungsalternierende aufwärtige Nystagmus, doch bei der Kopfhängelage erscheint er als Nystagmus diagonalis. Bei der Lagerungsprüfung tritt der feinschlägige Nystagmus mit Schwindelgefühl sehr aktiv auf.

b) Die ENG-Registrierung dessen Spontannystagmus.

c) Bei der Pürfung der Blickfolgebewegung ist eine deutliche Verfolgungsbewegungsstörung gezeigt.

doch bei der Kopfhängelage erscheint er als Nystagmus diagonalis. Bei der Lagerungsprüfung tritt der feinschlägige vertikale Nystagmus mit Schwindelgefühl sehr aktiv auf. Die Entstehungshemmung des optokinetischen Nystagmus (rechts $>$ links). Bei der Prüfung der Blickfolgebewegung ist eine deutliche Verfolgungsbewegungsstörung gezeigt (s. Bilder 2-a, b, c). Die Resultate des vertikalen Schreibversuchs sind günstig.

Ferner beim Decken des linken Auges erscheint der rechtswärtige horizontale und beim Decken des rechten Auges der linkswärtige horizontale Nystagmus. Der Nystagmus, der konstant erscheint und dessen Richtung sich kurzperiodisch nach links und rechts verändert, verschwindet nur indessen und wird ein latenter Nystagmus. 
Bei CAG-und VAG-Prüfungen sind keine besonderen pathologischen Befunde erhältlich.

Betrachtung: Der bei diesem Patienten erkenntbare kurzperiodisch unregelmässig richtungsalternierende horizontale spontane Nystagmus ist auf Grund dessen Erkrankungszustandes und Prüfungsresultate so zu betrachten, dass er wie beim ersten Fall mit der vasculär-pathologischen Veränderung im Wurm-Bereich verursacht wurde. Im Vergleich mit dem ersten Fall ist der Erkrankungszustand leichter, deshalb ist es nicht denkbar, dass sich das „Haematom" befindet. Als dessen Ursache ist eine Kreislaufstörung bzw. kleine Blutung vorzustellen. Aber bei diesem Fall muss die Beziehung zwischen dem obengenannten eigenartigen Nystagmus und latentem Nystagmus genug in Betracht gezogen werden. Doch unter Berücksichtigung der Tatsache, dass erst bei dem letzten Anfall der Patient Oscillopsie selbst merkte, und dass die Schwester des Patienten (Krankenschwester) erst nach dem Krankenhauseintritt den obenerwähnten spontanen Nystagmus bemerkte, ist es zu vermuten, dass der erstere ein mindestens mit diesmaligem Anfall in Verbindung stehender Nystagmus ist, und der letztere ein mit Astigmatismus und Strabismus convergens zusammenhängender Nystagmus ist, der wohl in hohem Masse angeborene Elemente hat.

Die aussergewöhnlichen Befunde wurden bei den morphologischen Untersuchungen wie VAG nicht erfasst. Jedoch wenn man auf den Störungsgrad in Rücksicht nimmt, oder, da diese Untersuchungen nur eine hilfsdiagnostische Bedeutung haben, bedeutet das nicht, dass die Resultate der anderen klinischen Prüfungen zu verneinen sind.

Fall III: K.I. 61 Jahre, männl. Arzt, dextromanuell.

Hauptsymptome: Kopfschmerzen, Schwindel, Entkräftensgefühl an Gliedern, Gleichgewichtsstörung.

Familiengeschichte: von Hypertonie und Apoplexie belastet.

Anamnese: vor 25 Jahren Pneumonie, seit 20 Jahren leidet er unter Hypertonie.

Status praesens: Seit ungefähr 10 Jahren leidet er manchmal, hauptsächlich am frühen Morgen, unter plötzlichen heftigen Hinterkopfschmerzen, Drehschwindel, Brechreiz, Erbrechen, Lähmungsgefühl und Schmerz an den beiden Armen. Die Häufigkeit dieses Anfalls ist 4-5 mal jährlich. Besonders bei einem Anfall vor 6 Jahren wurde der allgemeine Krampf mit obengenannten Anfällen belgeitet, und etwa eine Woche lang dauerte der Tremor an den beiden Armen.

Von 4. oder 5. Monaten an, nach diesem Anfall vor 6 Jahren, wankt er oft vorwärts und allmählich wird sein Gang gestört. Eine Zeitlang danach bekam er Anfälle nicht so oft, aber seit etwa zwei Jahren erscheint der Anfall wieder. Das blutdrucksenkende Mittel und Krampfmittel wurden eingenommen, doch wurde seine Krankheit nicht besser. Vor drei Monaten bekam er einen ziemlich heftigen Anfall und dabei entdeckte sein Freund, Arzt, den Nystagmus.

Prüfungsresultate: Blutdruck 165-112. Augenhintergrund, K-W II-Grad. Die beiderseitigen leichten Stauungspapillen sind erkennbar. Kein Befund, der das Dasein des Hippel-Lindau-Haemoangioms bestätigt, ist erhältlich. In der aufgerichteten Haltung Equilibrium Res Suppl. 1 
beim Augenschliessen fällt der Patient leicht hinterwärts. Beim Tretversuch hat er eine Falltendenz vor- und hinterwärts. Kein Kleinhirnsymptom. Auch kein Hirnnervensymptom. Der Sehnenreflex steigert sich beiderseitig ein bisschen. Kein pathologischer Reflex ist sichtbar. Der zerebrospinale Druck und dessen Eigenschaft sind normal.

Wenn der Patient ohne Brille nach vorne ansieht, ist der kurzperiodisch unregelmässig richtungsalternierende horizontale Nystagmus erkennbar. Beim Auf- und Niederblick wird dieser Nystagmus ein vertikaler Nystagmus in Unteraugenlidrichtung, beim Blick nach rechts und links wird er ein Nystagmus in rechten und linken Blickrichtung, und in beiden Fällen ist keine Veränderung der Richtung zu erkennen. Unter Benutzung der Leuchtbrille ist kein Nystagmus erkennbar. Bei der Lageprüfung ist der richtungsalternierende aufwärtige Nystagmus festzustellen, und auch bei der Kopfhängelage wird ein vertikaler Nystagmus veranlässt. Bei der Lagerungsprüfung ist ein feinschlägiger vertikaler Nystagmus sichtbar. Aber dabei klagte der Patient gleichzeitig über ein starkes Schwindelsgefühl. Die Entstehungshemmung des optokinetischen Nystagmus. Die Motorik der

Spontan-u.Blicknystagmus

Spontan-und Provokations-Nystagmus
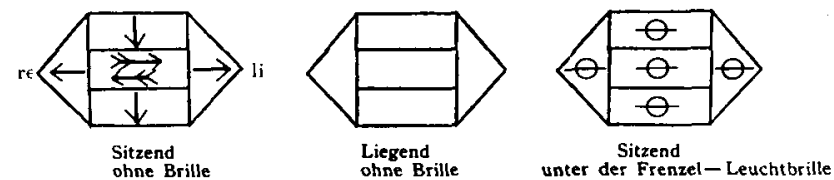

Lageprüfung

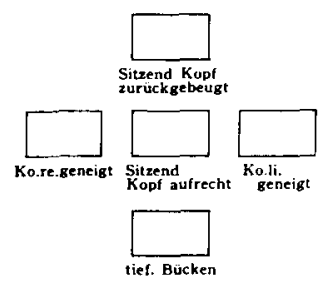

Lagerungsprüfung

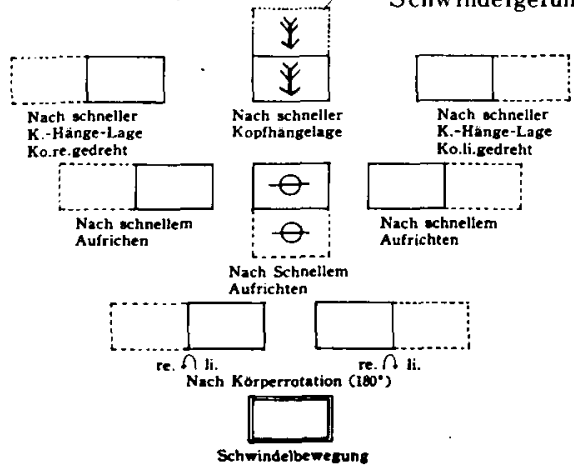

Abb. 3: K.l., $61 \hat{\jmath}$

Wir konnten fast dieselben Befunde wie bei ersten und zweiten Fällen feststellen. 
Blickzielverfolgung ist ziemlich gestört (s. Bild 3).

In CAG und VAG ist keine bemerkenswerte Veränderung sichtbar, aber bei PEG befindet sich ein symmetrisches Vergrösserungsbild der beiderseitigen Gehirnkammer und des Ventriculus tertius. Durch die Myodil-Ventrikulographie ist ein Befund, der vielleicht als leichter Schattendefekt im hinteren Bereich des Ventriculus quartus zu betrachten ist, erhältlich.

Operationsbefunde: Da auch der Patient wünschte, wurde die Kranitomie durchgeführt.

In der Kleinhirnhemisphäre wurde keine aussergewöhnliche Veränderung festgestellt, aber im Wurm wurde die Atrophie betrachtet und besonders im Unterwurm war schon deren Spur vorhanden. Jedoch im Bereich des Oberwurms über Mittelwurm war ein aussergewöhnlicher Vorwölbungsteil sichtbar. Durch die Punktion wurde ca. $1.5 \mathrm{cc}$ gelbe Flüssigkeit aspiriert. Beim Aufschneiden war ein kleines Blutgerinnsel erkennbar. Die Cystis wurde mit physiologischer Kochsalzlösung genug gespült und ein Teil der inneren Wand experimentell excidiert. Patho-histologisch war kein Befund des Haemoangioms feststellbar.

Betrachtung: Bei diesem Fall wurde der Anfall langperiodisch wiederholt. Es ist zu erfassen, dass zwischen diesen Anfällen und pathologischen Veränderungen am Wurm irgendeine Beziehung bestnad. Es scheint mir, dass es eine enge Kausalität mindestens zwischen dem Anfall vor drei Monaten und dieser krankhaften Veränderung des Wurms, ferner eigenartigem Nystagmus gibt. Aber ich kann leider nicht zu einer Folgerung darüber gelangen, ob das Atrophiebild, das im ganzen Wurm betrachtet wurde, mit den lange wiederholten Anfällen zusammenhängt, oder, ob diese Atrophie ein sogenanntes „Degenerationszeichen" ist und die Ursache dualistisch erklärt werden soll.

Fall IV: Y.M. 68 Jahre, weiblich, Hausfrau, dextromanuell

Hauptsymptom: Bewusstseinsstörungsanfall, Hinterkopfschmerzen, Lähmungsgefühl an der Gliedern, Schwindel, Körperschwandkungsgefühl, Oscillopsie und Schluterspannung.

Familiengeschichte: Die Eltern und auch beide Brüder sind an Apoplexie gestorben.

Anamnese: Als die Patientin 19 Jahre alt war, litt sie an Gelenkrheumatismus, mit 47 Jahren wurde sie wegen des Wurmfortsatzentzündung operiert, mit 49 Jahren wurde die Kastration des Ovarialcyste, mit 58 Jahren die Operation des Hämorrhoidalknoten und mit 65 Jahren die Operation des Zwölffingerdarmsgeschwürs durchgeführt.

Status praesens: Um 6 Uhr eines Morgens vor etwa anderthalb Jahre wurde die Patientin wach, klagte laut mit Ach und Weh über Schmerzen und wurde sofort bewusstlos (10 Tage dauerte diese Bewusstlosigkeit). Am Tag darauf wiederholte sie heftiges Erbrechen. Der Arzt wurde abgeholt und diagnostizierte als Apoplexie. Nach 10 Tagen, als sie wieder zum Bewusstsein kam, bekam sie einen starken Kopfschmerz. Sie wollte zur Toilette gehen, aber konnte nicht. Sie konnte auch Sitzhaltung nicht halten. Sie konnte mit anderen Leuten sprechen aber wegen des Ermündens verständigte sie sich schriftlich. Schreiben konnte sie gut. Die Glieder waren lahm. Die Schluckbehinderung erschien.

Während 6 Monate danach lag sie im Bett, und dazwischen versuchte sie vergebens aufzustehen und dabei bekam sie oft einen Drehschwindelanfall. Und einst fiel sie 
deswegen und ihr Gelenk der linken. Hand wurde gebrochen. Seit einem Jahr kann sie kriechend zur Toilette gehen. Seit diesem Zeit-Punkt erscheint beim Sehen ein Oscillopsie nach rechts und links und vor dem Halbjahr wird diese Erscheinung langsam besser. Das Körperschwankungsgefühl entsteht besonders stark beim Lagewechseln, aber auch bei Ruhelage ist es zu empfindèn.

Priffungsresultate: Blutdruck 172-114. Augenhintergrund K-W II-Grad. Keine Stauungspapille ist sichtbar. Kein Befund, der das Vorhandensein des Hippel-LindauHaemoangioms bestätigt, ist erhältlich. Romberg' Symptom ist stark positiv. Hypästhesie des linken Gesichts ist über ganzes Astbereich bemerkt. Der linke Hornhautreflex ist auch gefallen. Die linke Gehörschärfe ist geschwacht. Die calorische Erregbarkeit ist beiderseitig aktiv. Die Geschmacksschärfe der hinteren linken Seite der Zunge ist auch geschwächt. Die Herabsenkung des Würgreflexes ist erkennbar. Die Empfindungsherabsenkung der hinteren Wand des linken Rachens und die motorische Störung des linken Stimmbandes sind zu erkennen. Die Thermalgiestörung des rechten halben Körpers, tritt auf. Keine auffallende Reflexanomalie ist festgestellt.

Der spontane Nystagmus, Bricknystagmus, ferner Lagenystagmus sind nicht zu beobachten. Nur bei der Verwendung der Leuchtbrille, wenn die Augenstellung nach links gewechselt wird, erscheint der linkswärtige horizontale Nystagmus und dauert. Bei der Lagerungsprüfung, wenn der Körper der Patientin von der Sitzlage in Kopfhängelage schnell gewechselt wird, sind die feinschlägigen frequenten vertikalen Nystagmen aufeinander betrachtbar und gleichzeitig wird über Schwindelgefühl geklagt (s. Bild 4).

Die Entstehung des optokinetischen Nystagmus entspricht ihrem Alter. Bei der Blickzielverfolgungsbewegung kann man dasselbe sagen. In rechter VAG ist nichts ungewöhnliches zu merken. Links fehlt unterwegs plötzlich der Kontrast der Arterie c.i. posterior.

Betrachtung: Bei diesem Fall kann man auf Grund der klinischen Symptome mit Recht behaupten, dass es lateral-bulbäres Syndrom gibt. Im allgemeinen ist es sehr schwierig, röntgenologisch die ungewöhnliche Veränderung der Arteria c.i. posterior zu erweisen,

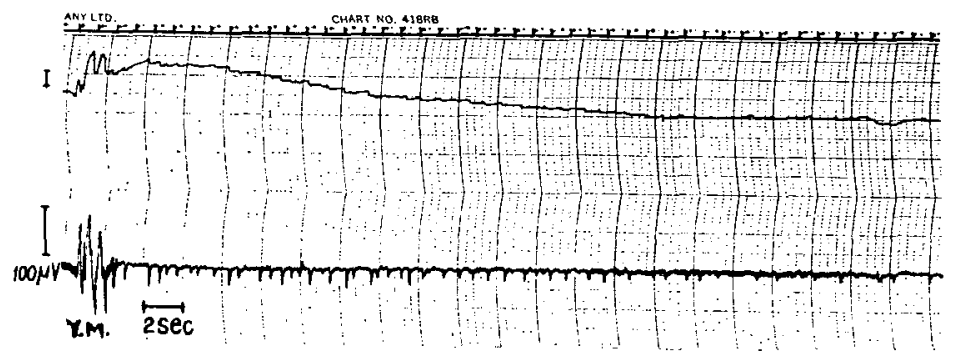

Abb. 4: Y.M., 68 우

Die ENG-Registrierung des vertikalen Lagerungsnystagmus. Nur bei der Lagerungsprüfung. wenn der Körper der Patientin von der Sitzlage in Kopfhängelage schnell gewechselt wird, sind die feinschlägigen, frequenten vertikalen Nystagmen aufeinander betrachtbar und gleichzeitig wird über Schwindelgefühl geklagt. 
aber bei diesem Fall ist es interssant, dass immer an der erkrankten Seite die Befunde, die die Ungewöhnlichkeit der Arteria c.i. posterior beweisen, gezeigt sind. Diese Befunde sind bedeutungsvoll, wenn man darauf Rücksicht nimmt, dass diese Arteria gewöhnlich in laterale Medulla oblongata, teilweisen Wurm und unterer Kleinhirnhemisphäre das Blut liefert. Es ist ein wichtiger und suggéstiver Befund, dass der Nystagmus sowie der Schwindel erst bei Lagerungsprüfung veranlässt und bei Wiederholungsprüfung wirderholt wurden. Es ist $\mathrm{zu}$ vermuten, dass im Oscillopsiezeitpunkt vom ungefähr Halbjahr, vom 6. Monate nach der Erkrankung irgendein Nystagmus entstand.

\section{DISKUSSION}

\section{Kleinhirnhaematom}

Das idiopathische Kleinhirnhaematom ist verhältnismässig selten und es ist so behauptet, dass sie nur etwa $10 \%$ von den idiopathischen Haematome im Schädel besetzt. 4, 8, 15, 17 Jedoch ist es nicht zu sagen, dass sie ein seltenes. Haematomn ist, wenn man denkt, dass das Volumenverhältnis des Gross- und Kleinhirns 12:1 ist.

Die Lokalisation des Haematoms im Kleinhirn ist aber meistens innerhalb der Hemisphäre und das Haematom, das sich nur im Wurm beschränkt befindet, ist selten. Die meisten Haematome befinden sich von dem Wurm bis in den Hemisphärenbereich erstdeckend. ${ }^{17}$ Wie bei unseren Fällen (Fall I und III) ist das Haematom, das nur im Wurm lokalisiert ist, ist ganz selten zu beobachten. Aber Goldsztain ${ }^{8}$ berichtet, dass er solches Haematom bei drei Fällen unter 21 Fällen von Kleinhirnhaematom erfuhr, und Dinsdale ${ }^{4}$ beobachtete dieselbe bei 3 Fällen unter 53 Fällen.

Als Ursache der idiopathischen Kleinhirnhaematoms steht die Hypertonie an der Spitze, und ferner sind Haemoangiom, haemorrhagische Diathese, Syphilis, Zuckerkrankheit, akute Infektionskrankheit, Arzneivergiftung, z. B. Vergiftung durch Alkohol usw. anzugeben. Bei dem Gesunden lässt sich angiomatöse Missbildung als deren Ursache leicht denken. ${ }^{1}$ Wie bei unserem Fall $\mathrm{I}$ ist die Epilepsie als eine deren Ursachen betrachtet $^{8}$, aber, wenn sich die Krise von derselben des epileptischen Anfalls unterscheidet, ist es ziemlich schwierig zu erfassen, wie weit die Epillepsie bei dem Krankheitszustand beteiligt ist.

Die Diagnose des idiopathischen Kleinhirnhaematoms ist nicht immer leicht, ${ }^{1,16}$ und es gibt viele Fälle, bei denen die Patienten innerhalb 24 Stunden nach der Blutung gestorben sind. Man sagt deshalb, es sei schwer, bei Lebzeit des Patienten zu diagnostizieren. Ferner ist diese Krankheit oft von den supratentorialen Erkrankungen kaum unterscheidbar, und wegen der Bewusstseinsstörung sind die Untersuchungen des Kleinhirnsymptoms meistens unmöglich. Es ist deshalb sehr schwer, die Lokalisation des Haematoms zu entscheiden. Infolgedessen sind dabei VAG und anderes röntgenologisches hilfsdiagnostisches Mittel anzuwenden. Aber durch solche morphologischen Untersuchungen ist das Haematom nicht erweislich, und es ist sehr selten, in früher Periode operiert zu werden.

Wird die Bewusstseinsstörung leicht, erscheint zuerst der Nystagmus, der eine der prüfungsfähigen Erscheinungen ist. In diesem Punkt ist die Untersuchung der Augenbe-

Equilibrium Res Suppl._l 
wegung, über die später in dieser Abhandlung erwähnt ist, sehr wichtig und wir haben erkennen können, dass er diagnostisch eine bedeutungsvolle Rolle spielt, wenn ein eigenartiger Nystagmus beobachtet wird.

\section{Das akute Wurm-Syndrom}

Es ist gesagt, dass der Wurm hauptsächlich von der Verästelung der Arterie c.i. posterior und zum Teil von der Arterie c. superior und medialis ant. die Blutversorgung erhält.

Als dessen klinisches Syndrom sind einige Syndrome wie Rumpfataxie, usw. angegeben, aber in wirklicher Klinik ist dessen Diagnose fast ummöglich, und es ist ein ungelöstes Gebiet. Die vier Fälle, die wir behandelten, zeigen alle eine akute Krise-Form, die ein charakteristischer Augenbewegungsbefund besitzen:

Nämlich, diese Krise beginnt immer mit dem plötzlichen (Dreh)—Schwindelanfall, Brechreiz und Erbrechen, sowie mit den heftigen Hinterkopfschmerzen und ferner ist der Fall mit dem Krampf festgestellt. Als Begleitungserscheinungen sind die Empfindungsungewöhnlichkeit der Extremität, besonders der beiden Armen und deren Entkräftensgefühl sowie die Bewusstseinsstörung erkennbar. Ausserdem ist es nicht selten, dass der Tremor dauert. In einem Fall wurde die Patientin früh am Morgen aus dem Schlafe erweckt. Mit dem Zeitverlauf wird ausser dem spontanen Schwindelanfall der von der Lage bedingte Schwindel mehr veranlässt.

Solcher Schwindelanfall hängt nicht von der Torsion der Halsgegend ab. In der Tat sind bei deisen Fällen keine Untersuchungsbefunde, die den Zervicalschwindel klarlegen, erhältlich. Ferner sind keine Prüfungsresultate erkennbar, die wie die Störung des Otolithenorgans das Dasein irgendeiner pathologischen Veränderung am Labyrinth bejahen. Das sogenannte „Kleinhirnsymptom“ an den Gliedern ist nicht zu beobachten. Der Blicknystagmus erscheint ja, aber er ist ziemlich anders als derselbe, der bei der reinen Hemisphärenstörung gezeigt wird, und sehr eigenartig ist. ${ }^{21}$ Der Fall IV zeigt deutlich das Syndrom des Unterwurms ${ }^{14}$, die Gleichgewichtsstörung mit Astasie tritt auf und die Patientin zeigt ferner Hypästhesie des einseitigen Gesichts und dissozierte Parästhesie des andern halben Körpers. Wie oben erwähnt, zeigt der Fall IV offenbar noch die Störung der vom Unterwurm beherrschten Zentralenausbreitung der Arteria c.i. posterior, während bei den Fällen, die Krampf, Bewusstseinsstörung, Tremor usw. zeigen, die Einflüsse der Arterie c. superior bzw. der penetrierenden Verzweigung der Arteria basilaris nicht zu verneinen sind.

Man kann keineswegs behaupten, dass die obengenannten Symptome nur bei den Wurmstörungen charakteristisch sind, aber die Ungewöhnlichkeit der Augenbewegung ist dabei sehr eigenartig und ein wichtiger Befund. Bei dem Geraderichtungsansehen oder lateralen Blick wird innerhalb sehr kurzer Zeit die Richtung unregelmässig nach rechts und links geweckselt und ist horizontal. Deshalb gibt es solche Fälle, bei denen die Oscillopsie subjektiv zu merken ist. Unter Benutzung der Leuchtbrille wird er gehemmt.

Bei der Lageprüfung wird der auftretende Nystagmus ein richtungsalternierender aufwärtiger und ferner bei Kopfhängelage ein vertikaler Nystagmus. Gleichzeitig wird das Schwindelgefühl beklagt. Dieser Nystagmus befindet sich fast konstant und ist bei 
jeden Prüfungen betrachtbar. Ausserdem, wie oft an demselben Tag wiederholt die Prüfungen auch versucht werden, ist er jedesmal zu erweisen und es ist festzustellen, dass er offenbar von dem labyrinthär unterschieden ist.

Kornhuber gibt besonders auf die Fälle, die den obengenannten vertikalen Nystagmus zeigen, acht und schlägt vor, diese Fälle das akute Unterwurm-Syndrom zu nennen. ${ }^{14}$

Jedoch, da oft bei den Störungen im Wurmbereich ausser dem Unterwurm sehr ähnlicher Krise-Vorgang sichtbar ist, möchte der Verfasser nicht nur auf Unterwurm beschränken, sondern ganzes Bereich umfassen und sie lieber das akute Wurm-Syndrom nennen.

3. Die eigenartige Augenbewegung beim akuten Wurm-Syndrom

Die klassische Einteilung des Kleinhirns, d.h. Archicerebellum oder Vestibulocerebellum, Paläocerebellum oder Spinocerebellum, und auch Neocerebellum oder Pontocerebellum, ist noch heute klinisch gültig. Darunter gehört Spinocerebellum dem Ober- und Unterwurm, und da die beiden bei Gleichgewicht oder Lageerhaltung des Körpers tief beteiligt sind, wird die Gleichgewichtsstörung veranlässt, wenn sie verletzt werden. Der Mittelwurm, besonders Folium oder Tuber, besitzt eine besondere Funktion und beteiligt sich an ,Blickmotorik“. ${ }^{19,}{ }^{20}$ Bei Katzen gibt es an Lobuli V, VI, VIIA viele Augenmuskelreceptoren und im VIIB sind sie zum Teil erkennbar. Infolgedessen nennt Hoshino sie „opthalmotropogene Zone".

Die anatomisch gefundene Klassifikation ${ }^{11}$ hat klinisch eine wichtige Bedeutung, z.B. beim Spätatrophien der Kleinhirnrinde beginnt die Atrophie mit den mit den Gliedern des Oberwurms zusammenhängenden Fasern. Bei der Hemisphärenstörung ist es dagegen bekannt, die Ataxie der Extremität oder Augen zu verursachen. ${ }^{21}$

Es ist gseagt, dass die centrifugalen Fasern der Kleinhirnrinden monosynaptisch auf den Kern des Kleinhirns eine Hemmungswirkung ausüben. Anderseits wirkt der Dachkern des Kleinhirns auf Formatio reticularis oder Vestibulariskern stimulierend und steht mit der horizontalen Augenbewegumgen im Zusammenhang. Infolgedessen treten umgekehrte Symptome bei der Störung der Rinden auf, im Vergleich mit der Kernstörung. Z.B., wenn die Rinde des Oberwurms der Katze einseitig entfernt wird, erscheint die Falltendenz in umgekehrter Richtung, und wenn der Dachkern an dieser Seite zerstört wird, fällt die betreffende Katze nach der gestörten Seite.

Bei der Störung von Nodulus ist die Erscheinung des Hemmungsausfalls sichtbar.

Wir möchten hier zwei neue Kleinhirnsymptome angeben und einige Betrachtungen darüber hinzufügen.

a. Das unregelmässige Burst von horizontalen Spontannystagmus, ,irregular burst of horizontal spontaneous nystagmus."

Bei den ersten drei Fällen, die wir behandelten, ist dieser Nystagmus zu beobachten. Wir haben zwei Fälle, bei denen durch die Operation das Dasein der pathologischen Veränderung im Wurm festgestellt wurde, aber dieses Symptom trat immer nach dem Anfall auf, und es gibt die Fälle, bei denen gleichzeitig die Oscillopsie subjektiv gemerkt wurde.

Wir sind deshalb zu einer Folgerung gelangen, dass die Störungen im Wurm oder in dessen Umgebung sich an der Erscheinung dieses Nystagmus beteiligen mögen. Der Fall,

Equilibrium Res Suppl. I 
von dem Takeyama und seine Mitarbeiter benachrichtigten, ${ }^{26}$ ist sowohl nach der KriseForm als auch nach den klinischen Symptomen den Fällen, die wir behandelten, äusserst ähnlich. Kornhuber warnt, dass der Gegenruck oder Treppenruck bei Gesunden für den pathologischen Nystagmus gehalten werden, wenn man die Prüfungen mit den geschlossenen Augen kurzperiodisch und nur wenig durchführt. Doch die Nystagmen, die bei unseren Fällen erkennbar sind, sind natürlich die davon unterscheidenen Nystagmen.

Wie schon erwähnt, gibt es als eine der Symptome der Wurm-Störung eine cerebelläre Fixationsstörung. Diese Störung ist hypometrisch und wird bei der grossen Blickakkommodationsbewegung sakkadierend. Die Störung, die bei der Prüfung der Blicfolgebewegung auftritt, ist ein Beispiel solcher Störungen.

Der eigenartige horizontale Nystagmus, den wir beobachteten, ist auch eine Störung der horizontalen Augenbewegung, deren Ursache auf dem Wurm beruht. Er ist auch die Ungewöhnlichkeit der Blickmotorik und unterscheidet sich wohl von dem blickparetischen Nystagmus, der bei Hirnstammstörung erkennbar ist. Experimentell ist in eine Periode solcher Nystagmus durch die Zerstörung des Dachkerns des Affens oder durch die Wurmstörung des Kaninchens berichtet. ${ }^{21}$

Also, ist solcher Nystagmus im Erscheinungsmechanismus dem sogenannten „Nystagmus alternans" identisch? Oder, soll dieser Nystagmus auch bei dem Namen „, Nystagmus alternans" genannt werden? Es ist notwendig, hier darüber zu betrachten.

Die Definition des Nystagmus alternans ist ganz verschieden, und je nach dem Gesichtspunkt der Gelehrten ist deren Behandlung auch sehr bunt. Mit anderem. Wort, es gibt unter dem Begriff der alternierenden Richtung des Nystagmus verschiedene Variationen, nämlich es gibt horizontale oder vertikale, regelmässig oder unregelmässig, konstant oder leichtveränderlich alternierende Nystagmen. Auch kommt dabei dessen Periode usw. in Frage. Deswegen gibt es unter den Definitionen der Forscher solche Theorien, die viele Fragepunkte in sich geschlossen sind, wie die Meinung von Kornhuber, „,der Nystagmus alternans ist ein horizontaler Nystagmus, dessen Periode beim Medianrichtungsblick mit geöffneten Augen nach rechts und links alternierend regelmässig gewechselt wird, unter der Nystagmus in einer Richtung dauert ungefähr 100 Sekunden ". ${ }^{13}$ Es gibt noch ferner Nystagmus vom anderen Typus; der Nystagmus, dessen Prozess sehr kurz ist, ${ }^{12}$ oder der Nystagmus mit kurzer und unregelmässiger Periode, über den Hosawa, ${ }^{10}$ Takeyama $^{26}$, Sakamoto ${ }^{18}$ usw. berichteten, ferner ein spezieller Nystagmus, der erst mit dem geschlossenen Augen auftritt, ${ }^{9}$ oder bei dem die Sehschräfe äusserst schlecht ist. Einige Forscher versuchen, nicht nur den Nystagmus, dessen Richtungsveränderung spontan ist, sondern auch den Nystagmus, dessen Richtung sich beim Verursachen der Schmerzempfindung oder beim transitorischen Wechseln der Blickrichtung erst verändert, in den Nystagmus alternans umzufassen.

Wenn die obengenannten verschiedenen Variationen alles als „Nystagmus alternans“ behandelt werden, kann man so sagen, dass der Fixationsnystagmus nach rechts und links oder der richtungsalternierende Lagenystagmus auch in Frage kommt, und meiner Meinung nach wird die Verwirrung um so mehr angerichtet. Deshalb nannte ihn Takeyama 
bei dem 18. Japanischen Vestibulum-Kongress ,,typusalternierenden Nystagmus“ und steht auf dem Standpunkt, dass er ihn zuerst mit Unterschied von dem typischen Nystagmus erfasst. Wenn die obengenannten verschiedenen Typen alles umgefasst werden, sind anderseits die Krankheiten, die für deren Ursache zu halten sind, ganz verschieden und bunt.

Nämlich, die folgenden Krankheiten sind anzugeben; angeborene, kopftraumatische, otogene, kleinhirnstörende, Hirngeschwulst-, Gehirnentzündungs-, multiplsklerotische, hirnkreislaufstörende, syphilitische, arachnoidesadhaesive, Zuckerkrankheit-, Erkrankungen. Infolgedessen gibt es auch verschiedene Meinungen über den Entstehungsmechanismus und das Krise veranlassende Moment dieses Nystagmus; Borries meint, dass er auf der Labyrinthfistel beruht, ${ }^{3}$ während Duensing usw. behauptet, er sei davon verursacht, dass durch das Hemmungslosmachen die von der Zentrumsstörung abhängige physiologische Postnystagmusperiode aufgetreten ist. ${ }^{\circ}$ Ohm führt Vestibulariskern als Enststehungslokalisation dieses Nystagmus an und behauptet, dass der Nystagmus dadurch verursacht wird, dass der Erregungszustand des Vestibulariskerns periodisch abwechselt, ${ }^{16}$ und Kornhuber berührt, dass seine Ursache in der beiderseitigen Störung des Formatio reticularis im Mittelhirn liegt. ${ }^{13}$ Bódó betrachtet, dass das Entstehungszentrum des Nystagmus alternans sich in der oberen Gegend des ersten vestibulären Neurons befindet. ${ }^{2}$ Neulich nimmt die Zahl der Berichte zu, dass der Nystagmus alternans durch das Kopftrauma festgestellt ist, aber wegen der Partikularität dieser Störung ist es aussergewöhnlich schwierig, daraus die verantwortliche Lokalisation zu schliessen. Jedoch ist die Kleinhirnwurm-Theorie nicht zu verneinen, da an der Wand des Ventriculus quartus, besonders in der Gegend des Tentriums durch dieses Trauma die hämorrhagische pathologische Veränderung leicht verursacht wird und in den Berichten als die Ursache die Kleinhirnstörungen verhältnismässig viel angegeben sind. Es scheint mir sehr unverständlich, dass trotzdem in meisten Theorien von heute ausser derselben von Stround ${ }^{25}$ als die Herdgegend dieses Nystagmus nur auf den Hirnstamm hingewiesen und keine Aufmerksamkeit auf den Kleinhirnwurm gerichtet ist.

Sei es falsch oder nicht, scheint mir vernünftig, dass die Benennung dieses Nystagmus, der seit Borries als Nystagmus alternans das Interesse der Forscher für sich allein behalten ist, aus den obengenannten Gründen nur in dem folgenden Fall gültig ist, : bei ,,dem horizontal-spontanem Rucknystagmus, dessen Richtung beim medianen Blick mit geöffneten Augen am hellen Ort periodisch nach rechts und links abwechselt, mit bestimmter PausePeriode regelmässig crescendo und decrescendo wiederholt, lange Jahre gehalten wird und bei den gedeckten Augen oder geschlossenen Augen, oder durch das Abnehmen der Fixationsblickfunktion wie beim Augenöffnen am dunkeln Ort beträchtlich gehemmt wird“' ${ }^{25}$, und dass man bei anderen Variationen, wenn deren Entstehungsmechanismus auch äusserst ähnlich sein mag, andere Benennungen, die die Eigenschaft jeder Variation ausdrucken, benutzt, oder sie anders einteilt, davon unterscheidet und anordnet.

Die Fälle, über die wir in dieser Abhandlung berichteten, zeigen auf die optokinetische Stimulatio eine typische Reaktionsform und noch dazu unterscheiden sie sich in vielen 
Punkten vom ,Nystagmus alternans".

Deshalb möchten wir vorschlagen, den von uns hier berichteten Nystagmus ,,das unregelmässige Burst von horizontalen Spontannystagmus, irregular burst of horizontal spontaneous nystagmus“ zu nennen und ihn von anderen Nystagmen zu unterscheiden.

Ferner, wenn unsere Schlussfolgerung, dass der von uns hier beschriebene eigenartige Nystagmus davon verursacht wird, dass die Störungen des mitteln Teils des Wurms, besonders im Teil des beiderseitigen Dachkerns auf den Formatio reticularis im Mittelhirn, Pons oder den Vestibulariskern einen Einfluss haben, richtig ist, mag dieser Nystagmus auch bei der Betrachtung des Entstehungsmechanismus des ganz typischen „Nystagmus alternans" und der anderen verschiedenen vestibulären Nystagmen sehr suggestiv und bedeutungsvoll sein.

b. Der feinschlägige, frequente, vertikale Lagerungsnystagmus mit Schwindelgefühl

Wie schon gesagt, ist es erwähnt, dass die centrifugalen Fasern der Rinde auf den Kleinhirnkern oder den Deiter' Kern eine Hemmungswirkung ausüben. Auch nach den Literaturen wirkt der Unterwurm, besonders Nodulus auf den Vestibulariskern hemmend. Wenn der Nodulus der Katze elektrisch stimuliert wird, ist der kalorische Nystagmus zu hemmen. Und wird der Unterwurm zerstört, wird der Lagenystagmus verursacht. ${ }^{7}$ Dieser Nystagmus verschwindet momentan doch, wenn man an dem inneren Ohr Procaine injiziert. Elektrophysiologisch hemmen die Purkinje' Zellen, die die centrifugalen Fasern ausbilden, die Neuron des Deiter' Kerns monosynaptisch. Daher ist das Dasein des Zentrummechanismus, der die Entstehung des reinen Lagenystagmus veranlässt, ausser dem Unterwurm jetzt noch nicht bekannt. Aber der Bericht, dass bei der beiderseitigen Dachkernsstörung der Katze wie am Wurm der vertikale Lagenystagmus verursacht wird, ist bemerkenswert. ${ }^{5}, 24$

Der vertikale, frequente Lage- oder Lagerungsnystagmus, der bei unseren Fällen beobachtet wurde, ist mit dem heftigen Schwindelgefühl verursacht und immer konstant entstanden, wie oft man die Prüfung auch wiederholt. Als die verantwortliche Gegend für Entstehung des vertikalen Nystagmus ist ausser dem Wurm noch verschiedene Teile zu beobachten. Jedoch aus obengenannten Gründen lässt sich diese Erscheinung leicht erklären, wenn man den Nystagmus von unseren Fällen für das Vestibularishemmungsausfallsympton, dessen Ursprung des Wurms, vor allem der Unterwurm hat, oder die Läsion der beiderseitigen Dachkerne, hält.

\section{ZUSAMMENFASSUNG}

Wir erführen vier Fälle, deren Symptome alle äusserst ähnlich waren und bei denen Schwindel, Kopfschmerz, Bewusstseinsstörung, Krampf usw. anfällig erschienen und wiederholt wurden. Bei 2 Fällen davon wurde das Dasein der Störung am Wurm durch die Operation festgestellt. Wir erörterten die Symptome von diesen Fällen und deren Prüfungsresultate und konnten die Ungewöhnlichkeit des Augenbewegung, die bei der WurmStörung sehr eigenartig ist, feststellen, so legte ich in dieser Abhandlung über die folgenden Angelegenheiten dar:

Equilibrium Res Suppl. I 
1. Über die Wurmstörung, deren klinische Diagnose bisher für unmöglich gehalten ist, beschrieb ich einige diagnostische Punkte und schlug vor, sie ,das akute Wurm-Syndrom" zu nennen.

2. Der eigenartige Nystagmus, der bei unseren Fällen beobachtet wurde, wurde dargelegt und „das unregelmässige Burst von horizontalem Nystagmus“ genannt. Der Verfasser gelangte zum Schluss, dass die Störung am Mittelwurm, vor allem am beiderseitigen Dachkern auf den Formatio reticularis im Mittelhirn, Pons und den Vestibulariskern einen Einfluss ausübte und diesen Nystagmus verursachte. Gleichzeitig äusserte ich meine Meinung über die Definition des seit langem bekannten "Nystagmus alternans" und zog einen Schluss, dass es vernünftig sei, bei der Betrachtung des Nystagmus die verschiedenen Variationen ausser dem typischen „Nystagmus alternans“ einzeln zu kalssifizieren und anzuordnen.

3. Ferner schloss ich darauf, dass der ,, feinschlägige vertikale Lagerungsnystagmus mit dem Schwindelgefühl" auf dem durch die Störung des Wurms, vor allem des Nodulus entstandenen Vestibularishemmungsausfall, oder die Läsion der beiderseitigen Dachkerne, der bei unseren vier Fällen zu erkennen war, beruhend verursacht wurde.

\section{SUMMARY}

Four cases with similar symptoms of fits consisting of vertigo, headache, disturbance of consciousness, and spasm, are presented. Two of them were confirmed by operation to have haemoatoma in vermis of cerebellum.

1) "The acute vermis syndrome", is defined and characterized in order to facilitate diagnosis in the future.

2) The characteristic nystagmus observed in these cases, are named "the irregular burst of spontaneous horizontal nystagmus". It is presumed that said nystagmus might be caused by disturbance in an intermediate part of vermis of cerebellum, particularly fastigial nuclei of both sides.

3) It is presumed that "vertical positioning nystagmus with vertigeous sensation" seen in the four cases might be caused by lack of vestibular repression due to the disturbance in the lower vermis.

\section{DANKWORT}

Zum Schluss möchte ich hier Herrn Prof. Syozo Kawamura meine herzliche Dankbarkeit dafür bezeigen, dass er freundlich mich beraten und diese Abhandlung durchsehen hat.

Ferner danke ich Herrn Dr. Hideo Naito, Direktor des Provinzial-Zentralkrankenhauses Ibaraki und den Ärzten dieses Hospitals, Herrn Dr. Buemon Sanbe, Oberarzt des KantoTeishin-hospitals sowie dessen Ärzten, Herrn Oberarzt Chikao Nagashima des Morokrankenhauses, und noch der Gruppe der Gleichgewichtsstörungsuntersuchung der HNO-Klinik an der Universität Tokio. 


\section{HAUPTLITERATUREN}

1) ARSENI, C. et al.: Cerebellar hematomas. J. Neurosurg. 16: 503-507, 1959.

2) Bódó, G. u.a.: Die elektronystagmographische Registrierung des Nystagmus alternans. Zbl. Ohrenheilk. 62: 74, 1959.

3) Borries, G. V. Th.: Vaskuläre Labyrinthfistelsymptome. Mschr. Ohr. Heilk. Wien 57: 443$481,1923$.

4) Dinsdale, H. B.: Spontaneous hemorrhage in the posterior fossa. Arch. Neurol. 10: 200217, 1964.

5) Dow, R. S. \& Morruzi, G.: The physiology and pathology of the cerebellum. The Univ. of Minesota Press, Mineapolis, 1958.

6) Duensing, F. u.a.: Reizwiederstandsfähiger (dominanter) und reizabhängiger Nystagmus alternans nach Hirntrauma. Arch. klin. exp. Ohr.-, Nas.-, u. Kehlk. Heilk. 168: 349-370, 1956.

7) Fernandez, C. et al.: Experimental observations on postural nystagmus. II. Lesions of nodulus. Ann. Otol. Rhin. \& Laryng. 69: 94-114, 1960.

8) Goldsztain, M.: The symptomatology of cerebellar haemorrhage. Acta Med. Pol. 4: 329$340,1963$.

9) Grawoushi, K. H. u.a.: Traumatisch bedingter Nystagmus alternans. HNO (Berl.) 13: 22-25, 1965 .

10) Hosawa, J. u.a.: Drei Beispiele des Nystagmus alternans. Otolaryng. (Tokyo) 36: 631635,1964

11) Hoshino, T.: Beiträge zur Funktion des Kleinhirnwurms beim Kaninchen. Acta oto-laryng. Suppl. 2: 1-72, 1921.

12) Katsumi, T. u.a.: Ein Beispiel des Nystagmus alternans. Pract. Otol. (Kyoto) 53: 1445$1453,1960$.

13) Kornhuber, H. H.: Der periodisch alternierende Nystagmus (Nystagmus alternans) und die Enthemmung des vestibulären Systems. Arch. klin. exp. Ohr.-, Nas.-u. Kehlk. Heilk. 174: 182-210, 1959.

14) Kornhuber, H. H.: Das akute Unterwurm-Syndrom beim Menschen. Ungarischer $O N O$ Sektionversamml. Budapest, $10: 24,1968$.

15) MC KIssock, W. et al.: Spontaneous cerebellar haemorrhage. A study of 34 consecutive cases treated surgically. Brain 83: 1-9, 1960.

16) Онм, J.: Der Nystagmus alternans. Zbl. Ohrenheilk. 13: 479, 1929.

17) Rey-Bellet, J.: Cerebellar hemorrhage. A clinicopathologic study. Neurology 10: 217-222, 1960.

18) Sакамото, M. u.a.: 12 Beispiele des Nystagmus alternans. Pract. Otol. (Kyoto) 61: 803-826, 1968.

19) Sakata, E. u.a.: Spontan-und Provokations-Nystagmus. Seine diagnostische Bedeutung bei Gleichgewichtsstörungen mit und ohne Schwindel. HNO (Berl.) 14: 289-298, 1966.

20) Sakata, E.: Das experimentelle Studium über den Spontan und Provokations-Nystagmus. Das Studium I: Das Zerstörungsexperiment der einseitigen Kaninchen-Labyrinth und des Kleinhirns. Jap. Jour. Otol. Tokyo 70: 385-394, 1967.

21) Sakata, E. u.a.: Das neurootologische Studium über die Kleinhirnstörung. Der I. Bericht: $\mathrm{Ob}$ die Störung der Kleinhirnhemisphäre durch die Prüfung der Augenbewegung diagnosti- 
ziert werden kann? Pract. Otol. (Kyoto) 61: 1642-1658, 1968.

22) Sakata, E. u.a.: Der Kleinhirntumor und Schwindelanfall, Méniére' Krankheit und deren Umkreis, V. Bericht. Pract. Otol. (Kyoto) 62: 1089-1100, 1969.

23) SAKata, E.: Pathohistologische Befunde eines sezierten klinischen Fall des periodisch alternierenden Nystagmus (N. alternans). im Druck.

24) Spiegel, E. A. \& Scala, N. P.: Positional nystagmus in cerebellar lesions. J. Neurophysiol. 5: $247,1942$.

25) Stround, M. H.: The otologist and the middline cerebellar syndrome. Laryngoscope 77 : 1795-1804, 1967.

26) Takeyama, I. u.a.: Ein Beispiel des Nystagmus alternans. Otolaryng. (Tokyo) 40: 291-297, 1968.

(Received February 11, 1971) 